\title{
Electrochemical Control of Growth Factor Presentation To Steer Neural Stem Cell Differentiation
}

\author{
Anna Herland, Kristin M Persson, Vanessa Lundin, Mats Fahlman, Magnus Berggren, \\ Edwin W H Jager and Ana I Teixeira
}

\section{Linköping University Post Print}

N.B.: When citing this work, cite the original article.

This is the authors' version of the original publication:

Anna Herland, Kristin M Persson, Vanessa Lundin, Mats Fahlman, Magnus Berggren, Edwin W H Jager and Ana I Teixeira, Electrochemical Control of Growth Factor Presentation To Steer Neural Stem Cell Differentiation, 2011, Angewandte Chemie International Edition, (50), 52, 12529-12533.

http://dx.doi.org/10.1002/anie.201103728

Copyright: Wiley-VCH Verlag Berlin http://www.wiley-vch.de/publish/en/

Postprint available at: Linköping University Electronic Press http://urn.kb.se/resolve?urn=urn:nbn:se:liu:diva-72171 


\title{
Electrochemical Control of Growth Factor Presentation to Steer Neural Stem Cell Differentiation
}

\author{
Anna Herland*, Kristin M. Persson, Vanessa Lundin, Mats Fahlman, Magnus Berggren, Edwin W. \\ H. Jager, Ana I. Teixeira*
}

The regulation of stem cell fate decisions builds on a dynamic interplay between extrinsic signals and cell intrinsic genetic and epigenetic programs. Growth factors (GFs) are a class of extrinsic signals critical for stem cell maintenance and differentiation. During embryonic development, the patterns of expression and activity of GFs are under precise temporal regulation. Importantly, in vivo many GFs are presented to cells anchored to components of the extracellular matrix (ECM) ${ }^{[1]}$. Several GFs, including Fibroblast Growth Factors (FGFs), strongly interact with heparin and heparan sulfate, common components of ECM proteoglycans ${ }^{[2]}$. Heparin binding stabilizes these GFs and is required for GF clustering and activation of membrane bound receptors, thereby modulating the signaling strength and duration ${ }^{[3]}$. Although growth factor (GF) anchoring reportedly affects fundamental aspects of GF signaling, in vitro stem cell differentiation protocols typically rely on sequential treatment with soluble GFs. Previous attempts to anchor GFs have commonly involved covalent immobilization or physical entrapment ${ }^{[4]}$, which are likely to affect their activity and cell accessibility. Importantly, temporal control in these approaches, if available, relies on enzymatic or hydrolytic degradation of a bulk material ${ }^{[5]}$, rendering these methods generally incompatible with adherent stem cell culture and affording poor temporal resolution of GF presentation.

[*] ((Dr. A. Herland, V. Lundin, Ass. Prof. A I Teixeira)) ((Cell and Molecular Biology))

((Karolinska Institute))

((von Eulers väg 3, 17177 Solna, Sweden))

Fax: (+46- 83393 80)

E-mail: anna.herland@ki.se, ana.teixeira@ki.se

((K M Persson, Ass Prof E W H Jager, Prof. M Berggren ((Department of Science and Technology, Organic Electronics))

((Linköping University))

((Bredgatan 33, 60174 Norrköping, Sweden))

Prof. M Fahlman

((Department of Physics, Chemistry and Biology))

((Linköping University))

((58183 Linköping, Sweden))

[**] ((The authors thank Prof Ola Hermanson (Karolinska Institutet) and lab members for cell culture facilities and assistance. The authors would like to acknowledge funding from the Swedish Research Council (VR) and the Swedish Foundation for Strategic Research (SSF; the OBOE center). V.L. was supported by a KID fellowship from the Karolinska Institute and A.H. was supported by a postdoctoral grant from VR. MB wishes to thank the Önnesjö foundation and Linköping University for financial support.))
To achieve an in vivo-like anchoring method of GFs with exact temporal control of their activity, we developed and evaluated an electroactive material that enables GF presentation and on-demand switch in GF bioavailability. The electroactive material is the conjugated polymer poly(3,4-ethylenedioxythiophene) PEDOT ${ }^{[6]}$, which incorporates anionic counter ions to counteract positive charges arising on the polymer backbone during oxidative electrosynthesis. Incorporation of heparin as a counter ion generated a material with good biocompatibility and stability, previously discussed in the context of neural prosthetics ${ }^{[7]}$. Electrochemical reduction of the pristine, as synthesized, material caused a decrease in ionic binding of heparin to PEDOT, hence facilitating surface exposure of heparin and GF binding to heparin. Electrochemical oxidation of PEDOT led to a more intimate association between heparin and the polymer, decreasing the bioavailability of the bound GF (Figure 1a,b).
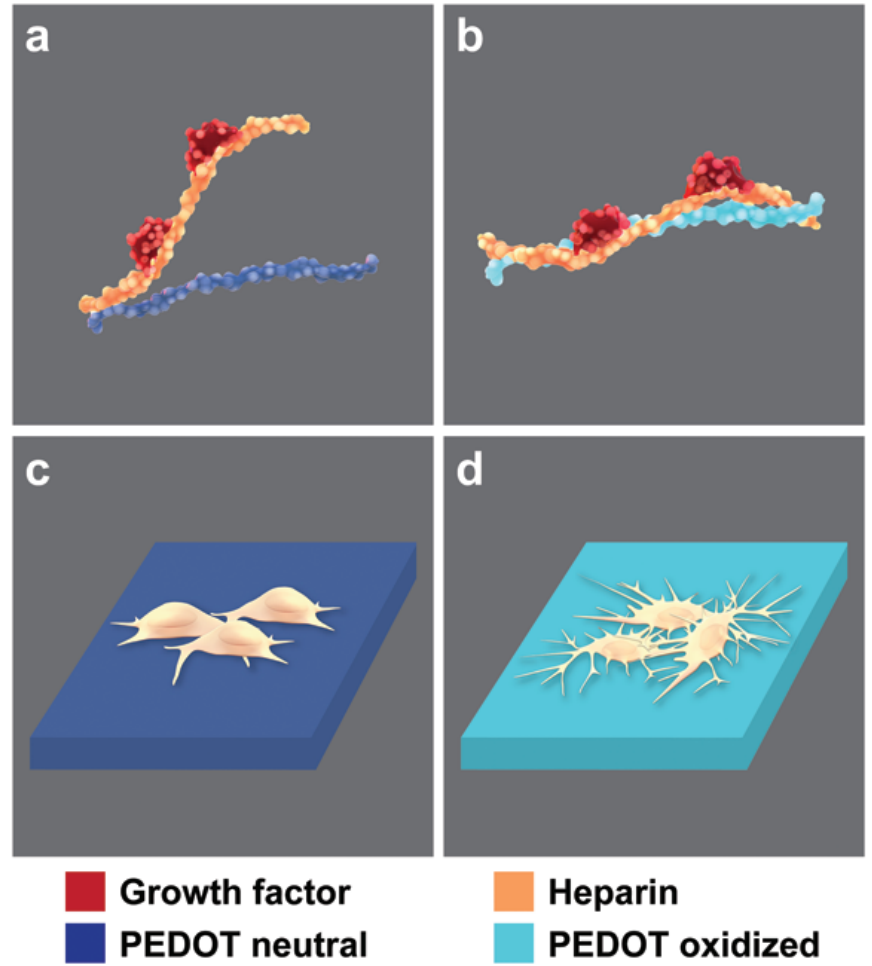

Figure 1. ((Electrochemical control of GF bioavailability to steer neural stem cell differentiation. a, b) Electrochemical oxidation of PEDOT leads to stronger electrostatic interactions between PEDOT and heparin, causing a decrease in the bioavailability of heparin binding GFs. c, d) Electrochemical oxidation of PEDOT induces NSC differentiation. a, c) Neutral PEDOT:heparin/GF. b, d) Oxidized PEDOT:heparin/GF. The top image illustrates the molecular dimension and the cellular response is shown in the bottom images. Note that structures are not drawn to scale. 
Embryonic neural stem cells (NSCs) are dependent on Fibroblast Growth Factor-2 (FGF2) to remain in a proliferative and undifferentiated state. Here we show that FGF2 anchored to PEDOT through heparin supports the proliferation and suppresses differentiation of NSCs cultured on PEDOT:heparin/FGF2 substrates. Remarkably, this process significantly stabilizes FGF2, eliminating the requirement for daily treatment of NSCs with soluble FGF2 ${ }^{[8]}$. Further, electrochemical oxidation of PEDOT decreases the bioavailability of FGF2, causing a reduction in cell proliferation and increased differentiation into neural cell types (Figure 1c,d). Together, these data demonstrate a method for temporal control of signaling cues to stem cells, a cornerstone for the development of devices for stem cell culture and cell therapy.

Electrosynthesis of 3,4-ethylenedioxythiophene (EDOT) in a Klexane ${ }^{\circledR}$-solution (pharmaceutical grade, low molecular weight heparin) onto a conducting substrate, yielded dark blue, semioxidized films ${ }^{[9]}$, carrying a net positive charge on the PEDOT chains balanced by negatively charged heparin molecules. Polymerization at $60 \mu \mathrm{A} / \mathrm{cm}^{2}$ for $1200 \mathrm{~s}$ resulted in a film thickness of about $400 \mathrm{~nm}$. Electrochemical reduction of the films rendered the polymer nearly neutral, from here on referred to as "neutral PEDOT". Electrochemical oxidation of neutral PEDOT yielded clear blue, fully oxidized films, denoted here as "oxidized PEDOT". The electrochemical characteristics of the material were evaluated with cyclic voltammetry. The redox peaks were at $-0.4 \mathrm{~V}$ and +0.3 $\mathrm{V}$, comparable to other PEDOT based materials (Figure S1). XPS $\mathrm{S}(2 \mathrm{p})$ core level spectra at various oxidation states were used to evaluate the presence of heparin on the surface of the films (Figure S2a-c). The $S(2 p)$ core level was fitted with two spin split doublets corresponding to sulfur atoms in PEDOT (low binding energy) and in heparin (high binding energy), respectively ${ }^{[10]}$. For the pristine film, the PEDOT-to-heparin sulfur ratio was $\sim 3.5$ (Figure S2a). For the neutral sample, the PEDOT-to-heparin sulfur ratio was significantly increased to $\sim 7.1$ (Figure S2b). This loss of heparin on the film surface is due to a decrease in the amount of positive charge of PEDOT upon reduction, resulting in weaker electrostatic interactions between the heparin molecules and PEDOT. The heparin molecules on the surface of the films can then be removed by blow-drying the samples with a stream of helium. These data are consistent with a model where there is an increased freedom of movement of the heparin molecules on the surface of the neutral films compared to the ionically attached molecules of the oxidized (and pristine) films. The heparin molecules deeper into the bulk are sterically hindered from escaping the film. Upon electrochemical oxidation of neutral PEDOT and blow-drying with helium gas, the PEDOT-to-heparin sulfur ratio was $\sim 4.4$ (Figure S2c). The relative increase in surface heparin in oxidized PEDOT compared to the neutral films is consistent with the presence of tighter interactions between heparin and PEDOT in oxidized films. To evaluate the accessibility of the anionic sulfate of heparin in the films, a Toluidine Blue (TB) assay was carried out ${ }^{[11]}$, omitting the blow drying procedure used for the XPS. In these experiments, the redox state of the surfaces was changed prior to the addition of TB. Oxidized PEDOT surfaces could only bind $\sim 53 \%$ of TB compared to neutral surfaces (Figure 2a), in accord with a previous report on TB binding to heparin in reduced and oxidized polypyrrole, a widely used conducting polymer ${ }^{[1]}$. Further, the decrease in TB binding to oxidized films was larger when PEDOT was reduced to the neutral state prior to oxidation than when pristine films were oxidized directly (data not shown), demonstrating the greater accessibility of the negative sulfate groups for TB binding in the neutral state.
Together, the XPS and TB assay data suggest that the sulfate groups of heparin are more available as interaction sites in neutral compared to oxidized PEDOT. In all subsequent experiments, polymer oxidation was preceded by reduction to neutral PEDOT to maximize accessibility of sulfate groups of heparin. This two-step procedure will hereafter be denoted simply as "oxidation". Electrochemical oxidation of neutral PEDOT is illustrated in the following reaction:

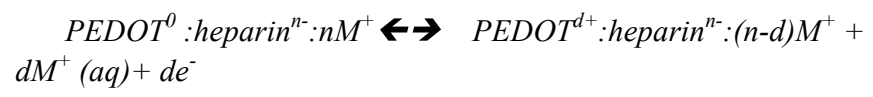

$\mathrm{M}^{+}$represents cations, $\mathrm{n}$ equals the total number of negative charges on heparin and $\mathrm{d}$ equals the induced positive charges on PEDOT.

Atomic force microscopy and goniometry were used to investigate the surface roughness and energy changes upon oxidation, respectively (see Supporting Information for description of methods). The surface roughness of neutral PEDOT, $15.88 \pm 3.84$ $\mathrm{nm}$, was not significantly different from that of oxidized PEDOT, $15.46 \pm 2.57 \mathrm{~nm}$ (Figure S3). Neutral PEDOT:heparin surfaces showed significantly lower water contact angles of $16^{\circ} \pm 2.6$, compared to oxidized surfaces, $21^{\circ} \pm 3.2(\mathrm{p}=0.05)$, consistent with the model of higher accessibility of charged species in the neutral state. a)

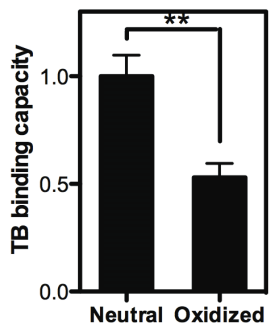

c)

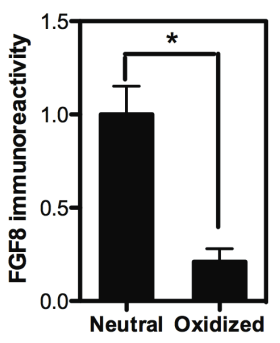

b)

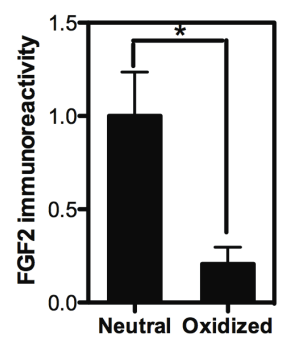

d)

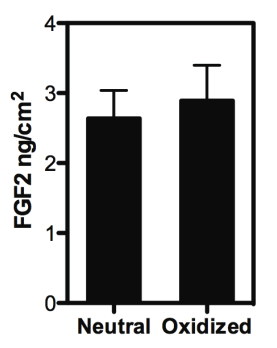

Figure 2. ((Characterization of PEDOT:heparin surfaces. a) Quantification of exposed heparin on neutral and oxidized PEDOT:heparin surfaces using Toluidine Blue assay, $n=6, p=0.005$; b) Antibody accessible FGF2 associated to neutral PEDOT:heparin incubated with $200 \mathrm{ng} \mathrm{ml}^{-1}$ FGF2 and left in a neutral state (Neutral) or oxidized after FGF2 incubation (Oxidized), $n=4, p<0.05$; c) Antibody accessible FGF8 bound to PEDOT:heparin on neutral surfaces incubated with $500 \mathrm{ng} \mathrm{m}^{-1}$ FGF8 (Neutral) or surfaces oxidized post FGF8 incubation (Oxidized), $n=3, p<0.05$; d) Total amount of anchored FGF2 on neutral and oxidized PEDOT:heparin surfaces incubated with $200 \mathrm{ng} \mathrm{ml}^{-1}$ of $\mathrm{I}-125$ labeled FGF2, quantified by gamma counting, $n=3$.))

Having characterized the electrochemically driven switch in heparin accessibility of PEDOT:heparin, we investigated the possibility of developing a switch for the presentation of heparin binding growth factors based on this method. In vitro maintenance of NSCs requires daily addition of soluble FGF2 to the culture. 
PEDOT:heparin films were reduced to the neutral state, to facilitate heparin interactions, and then incubated with FGF2 at various concentrations (Figure $2 \mathrm{~b}$ and Figure S4a,b). Following surface adsorption of FGF2, samples were coated with poly-ornithine and fibronectin, required for NSC culture. Finally, samples were either kept in a neutral state or were electrochemically oxidized in NSC culture medium. To demonstrate the generality of the procedure, we also used FGF8, another member of the FGF family of GFs with diverse roles in the regulation of the stem cells. Immunolabeling showed that oxidation of PEDOT:heparin/FGF2 resulted in a significant drop in the amount of antibody accessible FGF2. Oxidized PEDOT:heparin/FGF2 showed $\sim 20 \%$ of antibody accessible FGF2 compared to the neutral surface (Figure 2b). Reduction of the oxidized surfaces back to the neutral state did not reverse the drop in antibody accessibility of FGF2 observed after oxidation. Importantly, the oxidation conditions selected did not cause significant effects on the amount of accessible fibronectin (Figure S4c), as shown for conjugated polymer films oxidized for longer periods ${ }^{[12]}$. Accordingly, oxidized surfaces showed $21 \%$ of the amount of antibody accessible FGF8, compared to neutral surfaces (Figure 2c), validating the applicability of the method to other heparin binding GFs.

The observed decrease in detected FGF2 upon oxidation of PEDOT:heparin/FGF2 could originate from a loss of FGF2 from the film surface or from a conformational change, either in the protein structure or in the polymer film, hiding antibody epitopes. An assay with radiolabeled FGF2-I125 demonstrated that the amount of GF associated to the PEDOT:heparin remained the same on the neutral and oxidized surfaces (Figure 2d). These data demonstrate that FGF2 remains in the polymer film upon oxidation of PEDOT:heparin/FGF2. Importantly, heparin bound to FGF2 carries free sulfate groups available for interactions with other molecules [13]. Together, these data support a model where oxidation of the polymer results in stronger electrostatic interactions between heparin and PEDOT, while the association of FGF2 to heparin is maintained.

Next, we elucidated whether PEDOT:heparin/FGF2 can serve as an electrochemical switch for the proliferation and differentiation of NSCs. NSCs at passage 2 were seeded onto neutral or oxidized surfaces, as described above. After 4 days of daily treatment with soluble FGF2, NSCs cultured on neutral and oxidized PEDOT:heparin surfaces, showed similar numbers of live cells. These data show that the redox state of the polymer does not affect NSC proliferation when soluble FGF2 is present in the medium (Figure 3a,b). As expected, cultures where no FGF2 was added show low cell numbers, irrespective of the redox state of the surfaces. In contrast, neutral PEDOT:heparin/FGF2 surfaces supported significantly higher levels of NSC survival than oxidized surfaces. In fact, cell numbers on neutral PEDOT:heparin/FGF2 were at the same levels as on PEDOT:heparin surfaces where soluble FGF2 was added daily. Of note, the requirement for daily addition of soluble FGF2 for NSC maintenance stems from the short half-life of FGF2 in solution of only a few hours ${ }^{[14]}$. Therefore, presentation of FGF2 bound to PEDOT:heparin led to a stabilization of the biological activity of FGF2, effectively replacing the proliferative effects of soluble FGF2. Importantly, the oxidized PEDOT:heparin/FGF2 surfaces showed similar cell numbers to the neutral or oxidized PEDOT:heparin surfaces with no FGF2 added, suggesting that the function of surface bound FGF2 was effectively abrogated by the oxidation process (Figure $3 \mathrm{a}, \mathrm{b}$ ). On conventional tissue culture plastics (TCPS), cell numbers were six times higher where FGF2 was added daily compared to surfaces incubated with

FGF2 prior to cell seeding (Figure S5a,b), demonstrating minimal activity of adsorbed FGF2 on TCPS surfaces.

In NSC culture, removal of FGF2 initiates differentiation along the neuronal or glial lineages. Immunocytochemical staining for the astrocytic marker GFAP showed suppressed astrocytic differentiation on neutral and oxidized surfaces where FGF2 was added daily, as expected (Figure S6a). Similarly, no astrocytic differentiation was detected on neutral PEDOT:heparin/FGF2 surfaces, confirming the presence of active FGF2 on these surfaces. In contrast, on the oxidized PEDOT:heparin/FGF2 surfaces, the presence of differentiated astrocytes was evident (Figure S6a). RTQPCR further demonstrated significantly higher mRNA expression of GFAP on the oxidized surfaces compared to neutral PEDOT:heparin/FGF2 or surfaces where FGF2 was added daily (Figure S6b). The trend of increased differentiation on oxidized compared to neutral PEDOT:heparin/FGF2 was similar for neuronal differentiation (Figure S7a).

a)
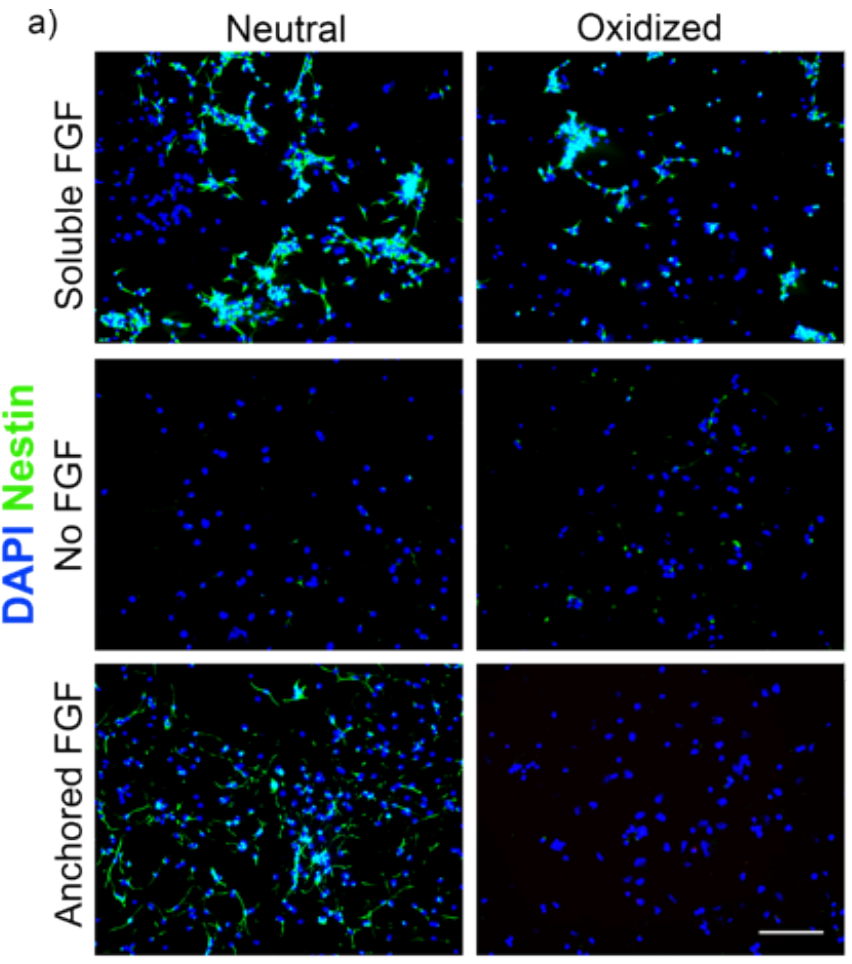

b)

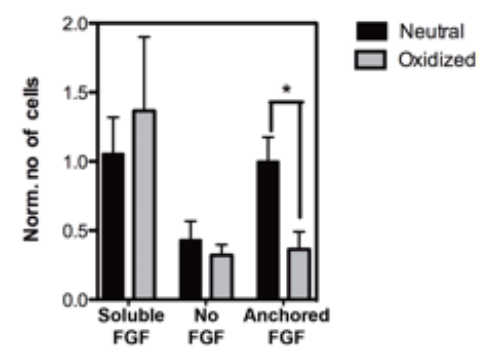

Figure 3. ((NSC culture on neutral and oxidized PEDOT:heparin /FGF2 surfaces demonstrating the biological activity of anchored FGF2. a) NSCs cultured for 4 days on PEDOT:heparin either kept in the neutral state or oxidized prior to cell seeding. FGF2 concentration was $10 \mathrm{ng} \mathrm{ml}^{-1}$ where FGF2 was added daily to the medium (Soluble FGF) and $200 \mathrm{ng} \mathrm{ml}^{-1}$ in samples pre-incubated with FGF2 (Anchored FGF). Control samples were neither treated with soluble nor preadsorbed FGF2 (No FGF). The scale bar corresponds to $150 \mu \mathrm{m}$. Green shows Nestin staining, a neural stem cell marker, and DAPI, a nuclear counterstaining, is shown in blue; b) Number of live cells on the surfaces $(n=3, p<0.05$, Student's t-test). )) 
Next, we examined the possibility to perform the oxidation process in situ during live cell culturing. NSCs were cultured overnight on neutral PEDOT:heparin/FGF2. Electrochemical oxidation was then performed or alternatively the system was kept as an open circuit. Comparison of the oxidized and open circuit samples where FGF2 was added in solution revealed that the oxidation process per se had no impact on the expression of GFAP, although the NSC viability was slightly decreased (Figure 4a). Importantly, the expression of GFAP clearly increased upon oxidation of PEDOT:heparin/FGF2 in situ (Figure 4a,b) and cells acquired an astrocytic morphology. A similar trend of increased neuronal differentiation upon oxidation PEDOT:heparin/FGF2 was observed (Figure S7b). Together, these results demonstrate that electrochemical switching of PEDOT:heparin/FGF2 decreases the bioavailability of FGF2, creating a defined onset of NSC differentiation.

\section{a)}
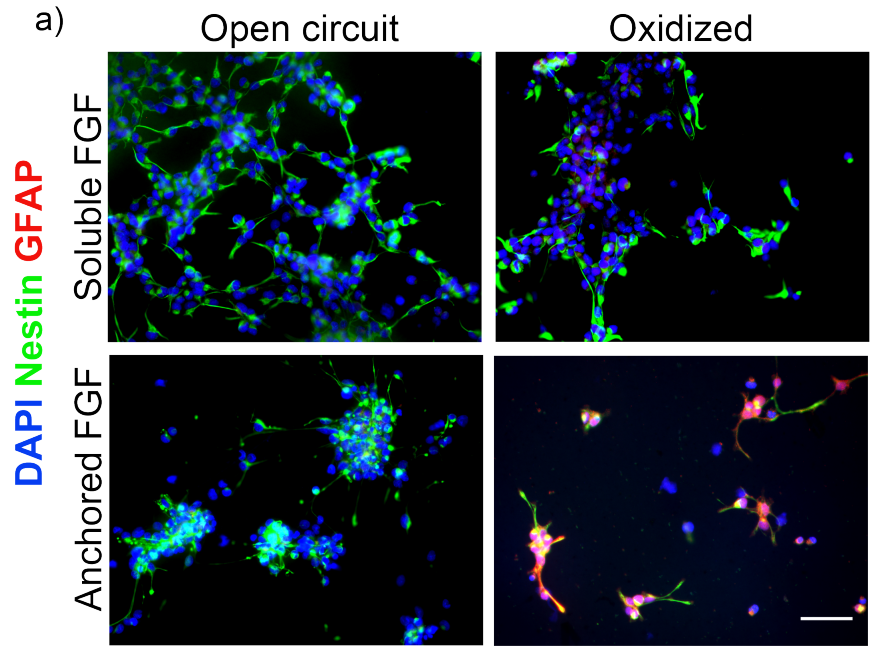

b)

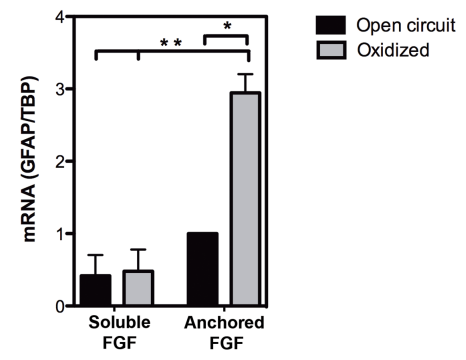

Figure 4. ((Oxidation of PEDOT:heparin/FGF2 surfaces decreases FGF2 activity during live cell culture. a) NSCs cultured for 4 days on PEDOT:heparin surfaces were oxidized with live cells or kept at open circuit. FGF2 concentration was $10 \mathrm{ng} \mathrm{ml}^{-1}$ for the daily treatment (Soluble FGF) and $200 \mathrm{ng} \mathrm{ml}^{-1}$ for the pre-incubated samples (Anchored FGF). The scale bar corresponds to $75 \mu \mathrm{m}$. Green shows Nestin, staining for NSCs, red is GFAP astrocytic staining and blue is DAPI nuclear counterstaing; b) RT-QPCR quantification of GFAP mRNA expression ( $n=2$, one way ANOVA).)

Here we describe a method that allows for presentation of heparin binding GFs to adherent stem cells. Association of GFs to heparin has been widely explored as a biomimetic strategy to immobilize GFs ${ }^{[15]}$. Importantly, in this study we demonstrate that the bioavailability of anchored GFs can be changed electrochemically through a straightforward oxidation step, offering precise temporal control of the stem cell state. This on-demand switch for GF bioavailability represents a significant improvement over methods relying on enzymatic or hydrolytic bulk degradation,

processes which are generally slow and afford little control over the onset of GF release.

We have validated a versatile tool, which offers a new dimension of control over cytokine presentation to cells. We suggest that it can be applied to a wide range of heparin binding GFs that undergo distinct temporal changes in activity, fundamental for cell regulation. Further, the approach has great potential to be tailored for 3D applications. Conducting polymers have previously been polymerized as coatings onto $3 \mathrm{D}$ scaffolds, composed of, for example, poly(ethylene terephthalate) (PET) ${ }^{[16]}$ or biodegradable polymers ${ }^{[17]}$. 3D scaffolds with active control of GF presentation are of true interest for stem cell therapy, which presently grapples with poor cell survival and limited control over differentiation of grafted cells ${ }^{[18]}$. Of note, we show stabilization of the activity of GFs anchored to PEDOT:heparin, a prerequisite for using this approach to control the stem cell microenvironment in vivo. We propose that the strategy presented in this study, in which cytokine bioavailability is regulated through the electrochemical properties of a conjugated polymer, has the potential to be a fundamental technique for basic studies of stem cell function as well as stem cell therapy.

For Materials and methods see Supporting Information.

Received: ((will be filled in by the editorial staff))

Published online on ((will be filled in by the editorial staff))

Keywords: Stem cells - Conjugated polymer - Growth factors · Heparin · Polymer

[1]
R. O. Hynes, Science 2009, 326, 1216-1219.

aI. Capila, R. J. Linhardt, Angew Chem Int Ed Engl 2002, -41, 412; bS. Tumova, A. Woods, J. R. Couchman, Int J. Biochem. Cell Biol. 2000, 32, 269-288.

L. Pellegrini, D. F. Burke, F. von Delft, B. Mulloy, T. L. Blundell, Nature 2000, 407, 1029-1034.

aK. Alberti, R. E. Davey, K. Onishi, S. George, K. Salchert, F. P. Seib, M. Bornhauser, T. Pompe, A. Nagy, C. Werner, P. W. Zandstra, Nat. Methods 2008, 5, 645-650; bN. Huebsch, D. J. Mooney, Nature 2009, 462, 426-432; cM. P. Lutolf, P. M. Gilbert, H. M. Blau, Nature 2009, 462, 433-441; dH. Yamazoe, Y. Murakami, K. Mizuseki, Y. Sasai, H. Iwata, Biomaterials 2005, 26, 5746-5754.

P. Tayalia, D. J. Mooney, Adv. Mater. 2009, 21, 3269-3285. aB. L. Groenendaal, F. Jonas, D. Freitag, H. Pielartzik, J. R. Reynolds, Adv. Mater. 2000, 12, 481-494; bA. J. Heeger, Angew Chem Int Ed Engl 2001, 40, 2591-2611; cR. H. Karlsson, A. Herland, M. Hamedi, J. A. Wigenius, A. Aslund, X. J. Liu, M. Fahlman, O. Inganas, P. Konradsson, Chem. Mat. 2009, 21, 18151821.

aM. Asplund, E. Thaning, J. Lundberg, A. C. Sandberg-Nordqvist, B. Kostyszyn, O. Inganas, H. von Holst, Biomedical Materials 2009, 4; bE. M. Thaning, M. L. M. Asplund, T. A. Nyberg, O. W. Inganas, H. von Hoist, Journal of Biomedical Materials Research Part B-Applied Biomaterials, 93B, 407-415.

aK. K. Johe, T. G. Hazel, T. Muller, M. M. DugichDjordjevic, R. D. G. McKay, Genes \& Development 1996, 10, 3129-3140; bA. I. 
Teixeira, J. K. Duckworth, O. Hermanson, Cell Res. 2007, 17, 5661.

[9] S. K. M. Jonsson, J. Birgerson, X. Crispin, G. Greczynski, W. Osikowicz, A. W. D. van der Gon, W. R. Salaneck, M. Fahlman, Synthetic Met 2003, 139, 1-10.

[10] G. Zotti, S. Zecchin, G. Schiavon, F. Louwet, L. Groenendaal, X. Crispin, W. Osikowicz, W. Salaneck, M. Fahlman,

Macromolecules 2003, 36, 3337

[11] B. Garner, A. Georgevich, A. J. Hodgson, L. Liu, G. G. Wallace, Journal of Biomedical Materials Research 1999, 44, 121-129.

[12] K. Svennersten, M. H. Bolin, E. W. H. Jager, M. Berggren, A. Richter-Dahlfors, Biomaterials 2009, 30, 6257-6264.

[13] S. Faham, R. E. Hileman, J. R. Fromm, R. J. Linhardt, D. C. Rees, Science 1996, 271, 1116-1120.
[14] A. Nur-E-Kamal, I. Ahmed, J. Kamal, A. N. Babu, M. Schindler, S. Meiners, Molecular and Cellular Biochemistry 2008, 309, 157166.

[15] E. S. Place, N. D. Evans, M. M. Stevens, Nat Mater 2009, 8, 457470.

[16] M. H. Bolin, K. Svennersten, X. J. Wang, I. S. Chronakis, A. Richter-Dahlfors, E. W. H. Jager, M. Berggren, Sens. Actuator BChem. 2009, 142, 451-456.

[17] J. Y. Lee, C. A. Bashur, A. S. Goldstein, C. E. Schmidt, 2009, 30, - 4335 .

[18] D. E. Discher, D. J. Mooney, P. W. Zandstra, Science 2009, 324, 1673-1677. 